\title{
Activation of $\alpha 7$ Nicotinic Acetylcholine Receptors Prevents Monosodium Iodoacetate-Induced Osteoarthritis in Rats
}

\author{
Yuan Liu ${ }^{b}$ Dongying Wu ${ }^{c}$ Fanglong Song ${ }^{\mathrm{a}}$ Chenlei Zhu ${ }^{\mathrm{a}}$ Yujian Huia \\ Qingcheng Zhu ${ }^{a}$ Jie $\mathrm{Wu}^{\mathrm{d}}$ Weimin Fan ${ }^{\mathrm{a}}$ Jun $\mathrm{Hu}^{\mathrm{a}}$
}

\begin{abstract}
aDepartment of Orthopedics, the First Affiliated Hospital of Nanjing Medical University, Nanjing, ${ }^{b}$ Department of Infectious Diseases, the First Affiliated Hospital of Nanjing Medical University, Nanjing, 'Department of Orthopedics, the Affiliated Hospital of Xuzhou Medical College, Xuzhou, China; 'Division of Neurology, Barrow Neurological Institute, St. Joseph's Hospital and Medical Center, Phoenix, AZ, USA
\end{abstract}

\section{Key Words}

Osteoarthritis • Nicotinic acetylcholine receptor - Monosodium iodoacetate - Chondrocyte $\bullet$ Rat

\begin{abstract}
Background/Aims: Although some evidence suggests that the prevalence of osteoarthritis $(\mathrm{OA})$ is lower in smokers compared to nonsmokers, the mechanisms of nicotine-induced protection remain unclear. Stimulation of the $\alpha 7$ nicotinic acetylcholine receptor $(\alpha 7-n A C h R)$ appears to be a critical mechanism underlying the anti-inflammatory potential of cholinergic agonists in immune cells. The inhibition of secreted inflammatory molecules and the subsequent inflammatory processes have been proposed as a novel strategy for the treatment of OA. The objective of the present study was to determine whether nicotine-induced protection in a monosodium iodoacetate (MIA) rat model of OA occurs via $\alpha 7-n A C h R$-mediated inhibition of chondrocytes. Methods: Both in vivo (MIA) and in vitro (MIA; Interleukin-1 $\beta$, IL-1 $\beta$ ) models of OA were used to investigate the roles and the possible mechanisms whereby $\alpha 7$ $n A C h R s$ protect against knee joint degradation. Multiple experimental approaches, including macroscopic, histological analysis, chondrocyte cell cultures, confocal microscopy, and western

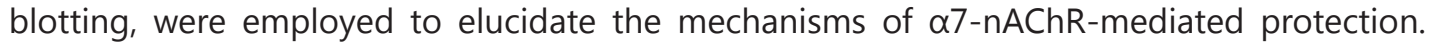
Results: Systemic administration of nicotine alleviated MIA-induced joint degradation. The protective effects of nicotine were abolished by administration of the $\alpha 7-n A C h R-s e l e c t i v e$ antagonist methyllycaconitine (MLA). In primary cultured rat chondrocytes, pretreatment with nicotine suppressed both p38, extracellular regulated kinase (Erk) 1/2 and c-Jun-N-terminal

Y.Liu and D. Wu contributed equally to this work.

Jun Hu, M.D., Ph.D.

Weimin Fan, M.D., Ph.D.

Department of Orthopedics, the First Affiliated Hospital of Nanjing Medical University, Guang Zhou Road 300, Nanjing 210029 (China)

Tel. +86 83714511; Fax +86 2583711066 , E-Mail junhu89@vip.sina.com
\end{abstract}

KARGER 125 
Liu et al.: Activation of a7-nAChRs Prevents MIA-Induced Osteoarthritis

kinase (JNK) mitogen-activated protein kinases (MAPK) phosphorylation and phosphorylated nuclear factor-kappa B (NF- $\kappa B$ ) p65 activation induced by MIA- or IL-1 $\beta$, and these effects were also reversed by MLA. Conclusion: Taken together, our results suggest that activation $\alpha 7-n A C h R s$ is an important mechanism underlying the protective effects of nicotine.

Copyright (c) 2015 S. Karger AG, Basel

\section{Introduction}

Osteoarthritis (OA), the most common type of arthritis affecting the elderly [1], dramatically impacts health care because of its negative effect on mobility. The extent of articular inflammation is normally associated with disease progression, pointing to articular damage as a contributor to this disease [2]. Recent evidence from both in vitro and in vivo studies suggests that chondrocytes can release and respond to a number of different cytokines, and are key active players during the disease process [3-5]. Interleukin (IL)-1 $\beta$ and tumor necrosis factor (TNF)- $\alpha$ are the foremost cytokines involved in the physiopathology of OA [6]. Consequently, drugs that inhibit secreted inflammatory molecules, such as proinflammatory cytokines, and their ensuing inflammatory induction, may provide understanding into enduring pathogenic injuries, and identifying molecular targets for new therapeutic interventions in $\mathrm{OA}$.

There is increasing evidence that nicotine employs anti-inflammatory and immunomodulatory effects on many cell types, such as T cells, B cells, dendritic cells, astrocytes and polymorphonuclear leukocytes, in the lung, spleen, liver, joints and the gastrointestinal tract [7]. The vagus nerve can modify immune responses and influence inflammation through an alpha7-nicotinic acetylcholine receptor ( $\alpha 7-n A C h R)$ dependent anti-inflammatory pathway [8]. Yu et al. reported that nicotine-induced modulation of adjuvant-induced arthritis (AIA) involves specific alterations in the disease-related cellular and humoral immune responses in AIA [9]. $\alpha 7$-nAChR knockout mice showed significant increases in both the incidence and severity of arthritis, and synovial inflammation as well as joint destruction were also demonstrated in murine collagen-induced arthritis (CIA) [10,11]. Evidence of the cholinergic anti-inflammatory pathway in the murine CIA model of rheumatoid arthritis or other arthritis models has been established, however, little is known about the role of $\alpha 7$ nAChRs in regulating pro-inflammatory cytokines in the pathophysiology of OA.

By injecting monosodium iodoacetate (MIA), a glyceraldehyde-3-phosphate dehydrogenase activity inhibitor, into a joint, one can rapidly induce OA-like lesions in both rodent and nonrodent models [12-15]. In rodents, this model yields cartilage lesions with loss of proteoglycan matrix and functional joint impairment much like human OA $[16,17]$.

Here, the main objective was the determination, using in vivo and in vitro models, of nicotine, acting on the $\alpha 7$-nAChRs, as an inhibitor of chondrocyte-mediated inflammation triggered by MIA, and potentially a cartilage degeneration preventer. This possible antiinflammatory mechanism, induced by nicotine, was further investigated using IL- $1 \beta$, the main proinflammatory cytokine involved in the pathophysiology of OA [6]. Moreover, we investigated whether $\alpha 7$-nAChR function is associated with modulation of phosphorylation of the mitogen-activated protein kinases (MAPKs) and members of the nuclear factor-kappa $\mathrm{B}(\mathrm{NF}-\kappa \mathrm{B})$ pathway.

\section{Materials and Methods}

Animals and treatments

All experiments were carried out in accordance with the guidelines of the National Institutes of Health for the Care and Use of Laboratory Animals (publication number 85-23, revised 1985) and the Society for Neuroscience Guidelines for the Care and Use of Animals in Neuroscience Research, and were approved by the Institutional Animal Care and Use Committee of Nanjing Medical University [18]. 
Liu et al.: Activation of a7-nAChRs Prevents MIA-Induced Osteoarthritis

Male Sprague-Dawley rats (2 months old, 175 to $200 \mathrm{~g}$ in weight) were used. All animals were housed in groups of five per cage under standard laboratory conditions with free access to food and water, constant room temperature $\left(22^{\circ} \mathrm{C}\right)$ and humidity $(50 \%$ to $60 \%)$, and a natural day/night cycle.

Nicotine, methyllycaconitine citrate (MLA), and MIA were dissolved in sterile saline (0.9\% $\mathrm{NaCl})$. Rats were randomly divided into groups as described below. All drugs were acquired from Sigma Chemical Co. (St. Louis, MO, USA).

The chronic MIA induction protocol was similar to that described previously [19]: $1 \mathrm{mg}$ of MIA in $50 \mu \mathrm{L}$ of sterile physiologic saline solution was injected once a week for 4 weeks into the right knees through the infrapatellar ligament. Rats were euthanized 1 week after final injection. Vehicle control rats were treated with saline. During nicotine treatment, rats were given intraperitoneal injections of nicotine $1 \mathrm{mg} / \mathrm{kg}$ once per day for 5 weeks ( 1 week before and throughout MIA administration). For all nicotine antagonism experiments MLA $1 \mathrm{mg} / \mathrm{kg}$ was injected $30 \mathrm{~min}$ before nicotine administration.

\section{Macroscopic analysis}

Macroscopic lesions were graded as follows: $0=$ normal appearance; $1=$ slight yellowish discoloration of the chondral surface; 2 = small cartilage erosions in load-bearing areas; $3=$ large erosions extending down to the subchondral bone; and $4=$ large erosions with large areas of subchondral bone exposure. Each of the chondral compartments of the knee (the femoral condyles, the tibial plateaus, the patella, and the femoral groove) was graded by two segregated observers who were blinded to the induction procedure. The four compartmental scores were combined (for a maximum possible score of 16) and the mean was determined for each group [20].

\section{Histological analysis}

Whole knee joints were fixed immediately in $4 \%$ paraformaldehyde, decalcified, and embedded in paraffin. Sagittal sections were stained with hematoxylin (HES) and toluidine blue. The severity of the OA lesions was graded according to Mankin's score [21] with minor modifications. This scale evaluates the severity of OA lesions based on proteoglycan integrity (scale $0-4$ ), cellular changes (scale $0-3$ ), collagen fiber integrity (scale $0-3$ ), invasion of the tidemark by blood vessels (scale $0-1$ ), bone modifications (scale $0-2$ ) and structural changes (scale $0-5$, where $0=$ normal cartilage structure and $5=$ erosion of the cartilage down to the subchondral bone) [22].

\section{Chondrocyte cultures}

Normal articular cartilage was obtained from Sprague-Dawley rats euthanized under anesthesia. After joint surgery, articular cartilage pieces were aseptically dissected from femoral head caps and chondrocytes were obtained by sequential digestion with pronase $(2 \mathrm{mg} / \mathrm{mL})$ and collagenase B $(1.5 \mathrm{mg} / \mathrm{mL})$ (Roche, Basel, Switzerland). The cells were washed twice in phosphate-buffered saline (PBS) and cultured to confluence in $75 \mathrm{~mm}^{2}$ flasks at $37^{\circ} \mathrm{C}$ in a humidified atmosphere containing $5 \% \mathrm{CO}_{2}$. The medium used was DMEM/Ham's F-12 supplemented with L-glutamine (2 mM), gentamicin $(50 \mu \mathrm{g} / \mathrm{mL})$, amphotericin B $(0.5 \mathrm{~g} / \mathrm{mL})$, and heat-inactivated fetal calf serum (FCS, 10\%). Chondrocytes were cultured under low FCS conditions $(10 \%, \mathrm{v} / \mathrm{v})$ and used at confluence [22].

\section{Staining with $\alpha$-bungarotoxin and confocal microscopy}

Primary cultured chondrocytes (as described above) were passaged into six-well tissue-culture plates at $5 \times 10^{5}$ cells per well, and then cultured for $24 \mathrm{~h}$. Thereafter, cultures were incubated with Alexa Fluor 488-conjugated $\alpha$-bungarotoxin $\left(2.5 \mu \mathrm{g} / \mathrm{mL}\right.$; B13422, Invitrogen Corp., Carlsbad, CA) at $4^{\circ} \mathrm{C}$ for $15 \mathrm{~min}$. Immediately after incubation, these cells were washed with PBS three times, and then fixed for $15 \mathrm{~min}$ in $4 \%$ paraformaldehyde in PBS at room temperature. After fixation, cells were washed once with PBS and then mounted for viewing under a laser scanning confocal microscope (Meta 710 Laser Scanning Microscope, Carl Zeiss Inc., Thornwood, NY) [18].

\section{Western blotting}

Cells were collected and homogenized in $200 \mu \mathrm{L}$ lysis buffer. After incubation for $20 \mathrm{~min}$ on ice, cell lysates were separated by centrifugation, and the protein concentration in the extracts was determined by the Bradford assay. Proteins in the cell extracts were denatured with SDS sample buffer and separated by $10 \%$ SDS-PAGE. Proteins were transferred to nitrocellulose membranes using a wet transfer unit (Miniprotean $®$ - 
Liu et al.: Activation of a7-nAChRs Prevents MIA-Induced Osteoarthritis

III; Bio-Rad Laboratories, Inc., Hercules, CA). The membranes were then incubated with 5\% BSA dissolved in Tris-buffered saline with Tween 20 (TBS-T, $10 \mathrm{mmol} / \mathrm{L}$ Tris-HCl, $150 \mathrm{mmol} / \mathrm{L} \mathrm{NaCl}$, and 0.1\% Tween 20, $\mathrm{pH}$ 7.5) at room temperature for $1 \mathrm{~h}$, washed three times, and incubated with different antibodies (p38, phospho-p38, extracellular regulated kinase1/2 [Erk1/2], phosphor-Erk1/2, the c-Jun-N-terminal kinase [JNK], phosphor-JNK and phosphor-NF-кB p65, 1:1000; Cell Signaling Technology Inc., Beverly, MA; $\alpha 7-$ nAChRs, 1:300, Santa Cruz Biotechnology Inc., Santa Cruz, CA) overnight at $4^{\circ} \mathrm{C}$. The following day the membranes were washed three times with TBS-T buffer, and incubated with secondary antibody for $1 \mathrm{~h}$, followed by four washes in TBS-T. Signal detection was performed with an enhanced chemiluminescence kit. The results were scanned using a gel imaging system (GelMax Imager; Ultra-Violet Products Ltd., Upland, CA) and measured using analyzing software (GelPro Analyzer software; Media Cybernetics, Inc., Bethesda, MD) [18].

\section{Statistical analyses}

All values are expressed as mean \pm standard error of the mean (SEM). Differences between means were analyzed using one-way or two-way analysis of variance (ANOVA) with time and treatment as the independent factors. When ANOVA showed significant differences, pairwise comparisons between means were further analyzed using the Newman-Keuls post hoc test. In all analyses, significance was set at $P=0.05$ [18].

\section{Results}

Nicotine acting via $\alpha 7-n A C h R$ s prevents MIA-induced cartilage degradation in rats.

To examine the effects of nicotine on cartilage degradation in rats, macroscopic and Mankin's scores of the knee joints acquired at the conclusion of the experiment were assessed. Intra-articular injection of $1 \mathrm{mg}$ of MIA induced knee joint deterioration, as evidenced by the cartilage on the central load-bearing areas of each articular surface was thin and yellowish, with focal erosions of the femoral condyles and tibial plateaus, as can be observed from the macroscopic score (Fig. 1A) [11, 23]. HES and toluidine blue staining revealed major remodeling of subchondral bone, loss of the hypertrophic chondrocyte layer and a decrease in cellularity in the MIA group (Fig. 1B, C). Nicotine (1 mg/kg) suppressed increases in MIAinduced macroscopic and Mankin's scores $(P<0.01)$ compared with MIA treatment alone. Interestingly, the protective effect of nicotine against MIA-induced cartilage degradation was reversed by pretreatment with MLA $(1 \mathrm{mg} / \mathrm{kg})$ (Fig. 1D, E) $(P<0.01)$. Notably, there were significant differences between the control group and the experimental group treated with both MIA and nicotine $(P<0.05)$, implying that although MLA could entirely invert the protective effect of nicotine against MIA-induced cartilage degradation, nicotine cannot fully prevent the MIA-induced cartilage degradation effects. No significant cartilage degradation changes were seen in mice treated with nicotine and MLA alone $(P>0.05$ compared with controls, data not shown).

\section{Expression of the $\alpha 7-n A C h R$ subunit in chondrocytes}

To further examine the hypothesis that nicotine-induced cartilage fortification in an MIA rat model is mediated through its actions on $\alpha 7-n A C h R s$ on chondrocytes, the expression of $\alpha 7-n A C h R s$ was evaluated using primary cultured chondrocytes. The $\alpha 7-n A C h R$ subunit protein was identified by western blotting in primary cultured cells (Fig. 2A). Furthermore, to evaluate whether $\alpha$-bungarotoxin, an $\alpha 7$-nAChR subunit-selective blocker, could bind to this receptor, primary chondrocytes were stained with Alexa Fluor 488 conjugated $\alpha$-bungarotoxin. Strong binding of $\alpha$-bungarotoxin was detected on the chondrocyte surface (Fig. 2B).

Nicotine acting via $\alpha 7-n A C h R s$ suppresses $p 38$, Erk1/2 and JNK MAPK activation induced by MIA in chondrocytes

MAPKs collectively constitute crucial signaling molecules in the development of inflammation. Phosphorylation of MAPKs leads to the commencement of signal cascades 
Fig. 1. Evaluation of nicotine-mediated protection against MIA-induced knee joint degradation by macroscopic and histological analysis. (A) Representative macroscopic photographs of tibial plateaus of the rat knee joints. (B) Photomicrographs showing representative hematoxylin staining of the rat knee joints. (C) Photomicrographs showing representative toluidine blue staining of the rat knee joints. (D) Semiquantitative macroscopic scores for joint destruction. (E) Semiquantitative Mankin's score for joint destruction. Data are presented as mean \pm SEM $(\mathrm{n}=6) .{ }^{*} P<0.05$ and ${ }^{* *} P<$ 0.01 vs. the control (saline

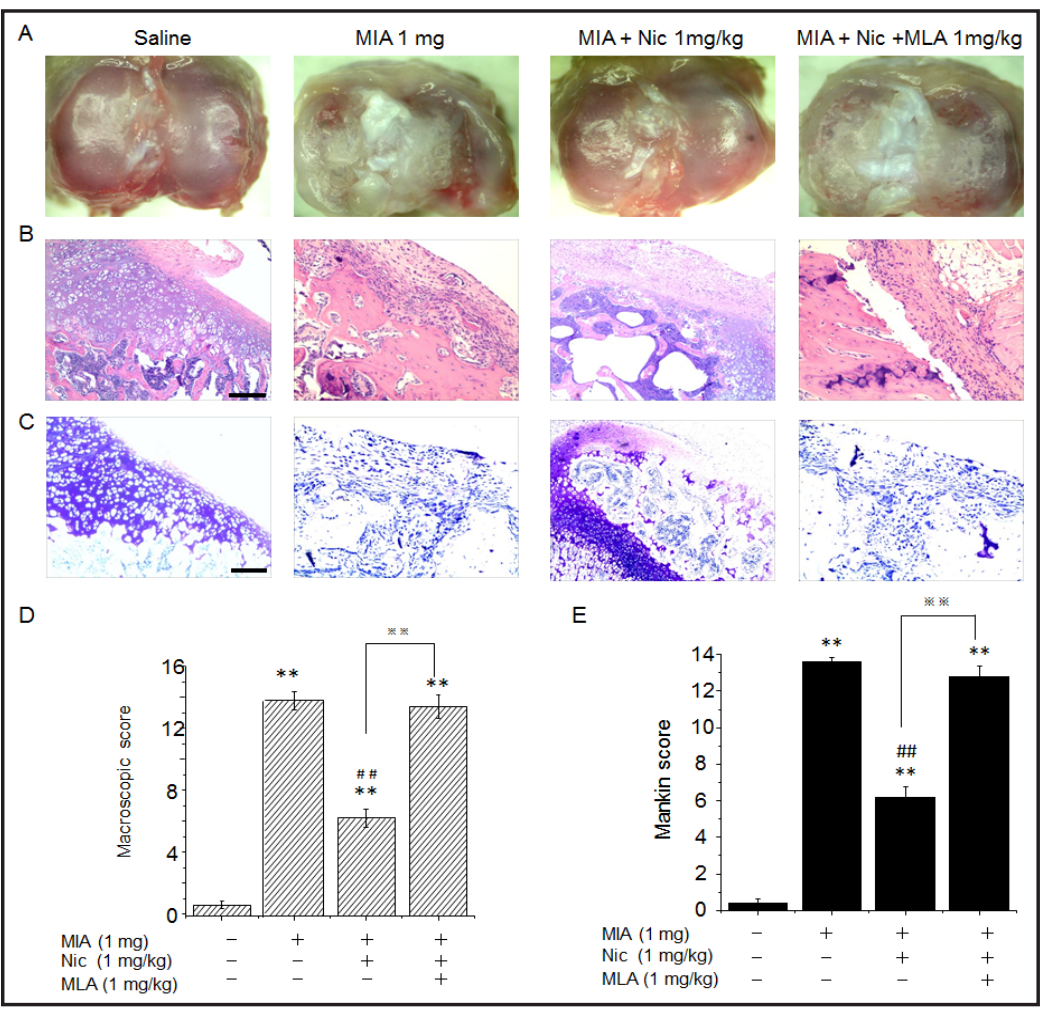
alone) group; $\# \# P<0.01$ vs. the MIA treatment group. ${ }^{*} * P<0.01$ between the MIA + nicotine treatment group and the MIA + nicotine + MLA treatment group. Scale bar: $200 \mu \mathrm{m}$.

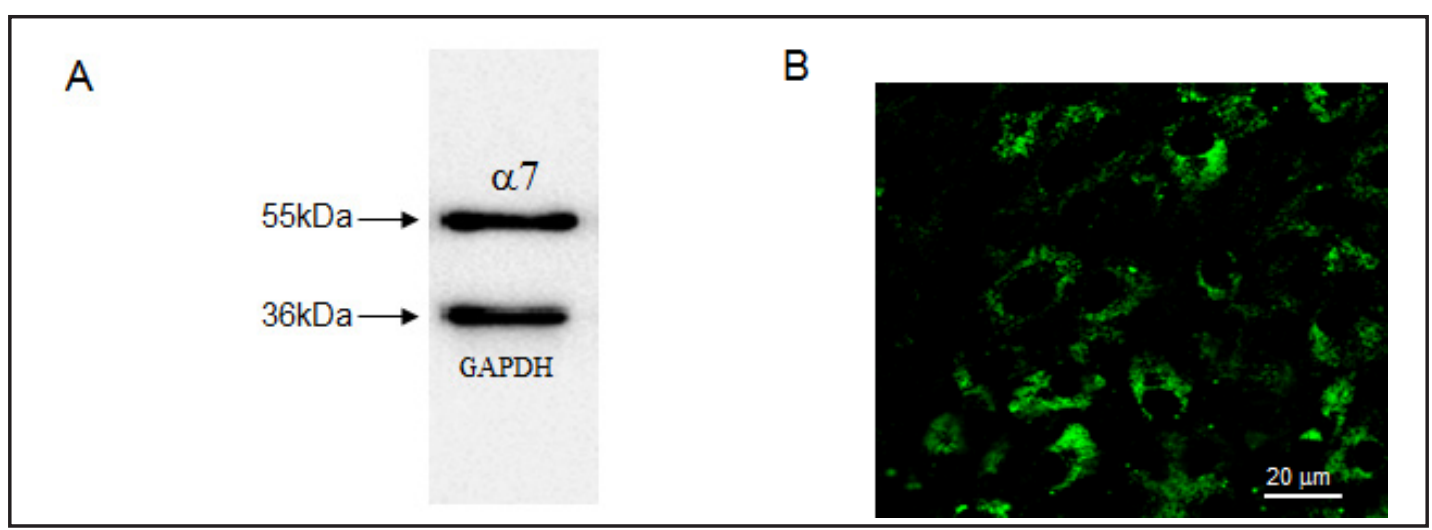

Fig. 2. $\mathrm{nAChR} \alpha 7$ subunit expression in primary chondrocyte cell cultures. (A) Western blot of $\alpha 7 \mathrm{nAChR}$ protein in primary cultured chondrocytes. (B) Primary rat chondrocytes were stained with Alexa Fluor 488 conjugated $\alpha$-bungarotoxin and viewed by fluorescent confocal microscopy. Scale bar: $20 \mu \mathrm{m}$.

that controls the creation of a variety of pro-inflammatory factors such as TNF- $\alpha$ in chondrocytes [24-26]. In the current study, we considered whether nicotine abolished the MIA-induced phosphorylation of p38, Erk1/2 and JNK MAPKs in chondrocytes. MIA treatment $(10 \mu \mathrm{mol} / \mathrm{L})$ induced rapid and transient phosphorylation of $\mathrm{p} 38$, Erk1/2 and JNK, reflecting their activation, and peak levels of phosphorylated p38, Erk1/2 and JNK occurred after $2 \mathrm{~h}$ (Fig. 3A, B, C). These outcomes imply that p38, Erk1/2 and JNK MAPKs are activated in response to MIA stimulation in chondrocytes. Then, we investigated the effects of nicotine on MIA-induced p38, Erk1/2 and JNK MAPK phosphorylation. Pretreatment with $10 \mu \mathrm{mol} / \mathrm{L}$ nicotine attenuated MIA-induced increases of phosphorylated p38, Erk1/2

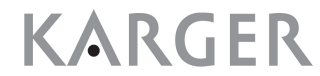


Fig. 3. The effects of nicotine on MIA-induced p38, Erk1/2 and JNK MAPK activation in chondrocytes. MIA increased p38, Erk1/2 and JNK MAPK phosphorylation, indicative of their activation. $(A, B, C)$ Chondrocytes were treated with $10 \mu \mathrm{M}$ MIA for various lengths of time as indicated. $(\mathrm{D}, \mathrm{E}, \mathrm{F})$ Chondrocytes were pretreated with nicotine $(10 \mu \mathrm{M})$ in the absence or presence of MLA (10 nM) for $30 \mathrm{~min}$ and then challenged with MIA $(10 \mu \mathrm{M})$ for $30 \mathrm{~min}$. After treatment, chondrocytes were harvested and levels of phosphorylated p38 (D), Erk1/2 (E) and JNK (F) MAPKs were analyzed. Lower: representative blots are shown. Upper: densitometric analysis of the phosphorylated forms of p38, Erk1/2 and JNK MAPK. Data are presented as the mean \pm SEM of four independent experiments. ${ }^{*} P<0.05$ and ${ }^{* *} P<0.01$ vs. the control (saline alone) group; \#\#P<0.01 vs. the MIA treatment group. $* * P<0.01$ between the MIA + nicotine treatment group and the MIA + nicotine + MLA treatment group.

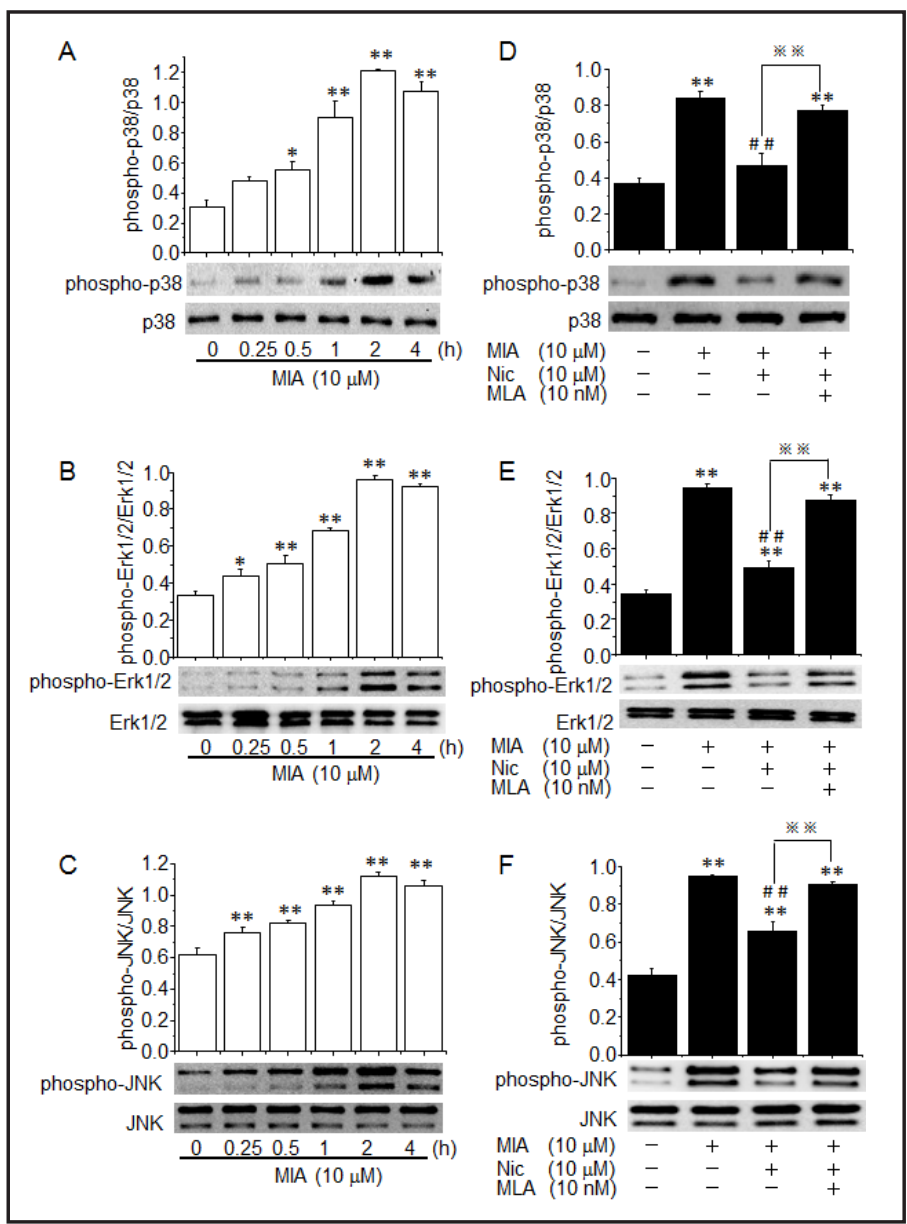

and JNK (Fig. 3D, E, F). Furthermore, the attenuating effects of nicotine on p38, Erk1/2 and JNK phosphorylation were completely blocked by $30 \mathrm{~min}$ of pretreatment with MLA at $10 \mathrm{nmol} / \mathrm{L}$. No significant changes of phosphorylated p38, Erk1/2 and JNK were seen in chondrocytes treated with nicotine and MLA alone ( $\mathrm{P}>0.05$ compared with controls, data not shown). Collectively, These data infer that nicotine, acting on the $\alpha 7$-nAChRs, suppresses MAPK signal transduction, thereby influencing inflammation systems.

Nicotine acting via $\alpha 7-n A C h R s$ suppresses $p 38$, Erk1/2 and JNK MAPK activation induced by $I L-1 \beta$ in chondrocytes

Amongst the proinflammatory cytokines implicated in OA, IL1 $\beta$ is believed to be the foremost in cartilage degradation [6]. We explored the possibility that nicotine also regulated IL1 $\beta$-induced MAPK signaling transduction. Chondrocytes were treated with IL1 $\beta$ at $10 \mathrm{ng} / \mathrm{mL}$ for different lengths of time to determine the extent of MAPK activation after IL1 $\beta$ stimulation. Treatment with IL1 $\beta$ also resulted in rapid and transient phosphorylation of p38, Erk1/2 and JNK, with peak levels also occurring at 30 min (Fig. 4A, B, C). These results suggest that $\mathrm{p} 38, \mathrm{Erk} 1 / 2$ and JNK are triggered in reaction to IL1 $\beta$ stimulation in chondrocytes. We then investigated whether nicotine could regulate IL1 $\beta$-induced p38, Erk1/2 and JNK phosphorylation. Pretreatment with nicotine at $10 \mu \mathrm{mol} / \mathrm{L}$ suppressed IL$1 \beta$-induced increases in phosphorylated p38, Erk1/2 and JNK (Fig. 4D, E, F). Additionally, the suppressive effects of nicotine on $\mathrm{p} 38, \mathrm{Erk} 1 / 2$ and JNK phosphorylation were entirely reversed by MLA (10 nmol/L). These data suggest that nicotine, through its actions on $\alpha 7-$ nAChRs, also inhibits IL1 $\beta$-induced chondrocyte activation via inhibition of MAPK signal transduction. 
Fig. 4. The effects of nicotine on IL1 $\beta$-induced p38, Erk1/2 and JNK MAPK activation in chondrocytes. IL1 $\beta$ also increased p38, Erk1/2 and JNK MAPK phosphorylation, reflecting their activation. $(A, B, C)$ Chondrocytes were treated with $10 \mathrm{ng} / \mathrm{mL}$ IL1 $\beta$ for various lengths of time as indicated. (D,E,F) Chondrocytes were pretreated with nicotine $(10 \mu \mathrm{M})$ in the absence or presence of MLA (10 nM) for $30 \mathrm{~min}$ and then challenged with IL1 $\beta$ (10 ng/mL) for $30 \mathrm{~min}$. After treatment, chondrocytes were harvested and levels of phosphorylated p38 (D), Erk1/2 (E) and JNK (F) MAPKs were analyzed. Lower: representative blots are shown. Upper: densitometric analysis of the phosphorylated forms of p38, Erk1/2 and JNK MAPK. Data are presented as the mean \pm SEM of four independent experiments. ${ }^{*} P$ $<0.05$ and ${ }^{* *} P<0.01$ vs. the control (saline alone) group; $\# P<0.05$ and $\# \# P<0.01$ vs. the IL1 $\beta$ treatment group. ${ }^{*} P<0.05$ and ${ }^{*} P<<$ 0.01 between the IL1 $\beta+$ nicotine treatment group and the IL1 $\beta+$ nicotine + MLA treatment group.

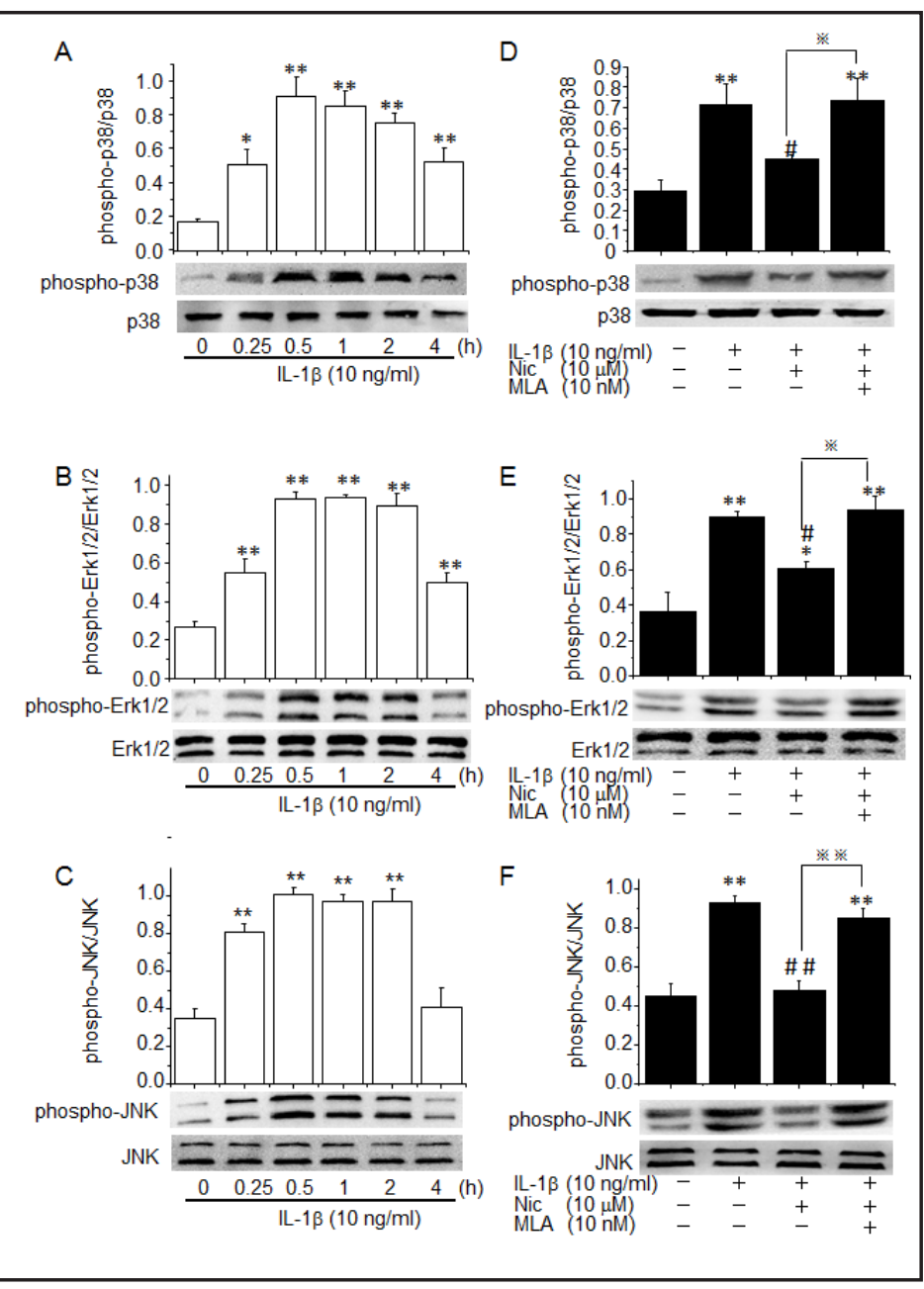

Nicotine or MLA administration alone did not significantly affect p38, Erk1/2 and JNK phosphorylation in chondrocytes ( $\mathrm{P}>0.05$ compared with controls, data not shown).

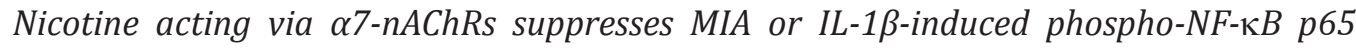
activation in chondrocytes

To further examine the mechanisms of nicotine-mediated suppression of inflammatory responses in MIA or IL1 $\beta$-activated chondrocytes, we focused on the activation of NF- $\kappa B$, a family of transcription factors related to inflammatory responses and cartilage degradation. The results suggest that treatment with MIA at $10 \mu \mathrm{mol} / \mathrm{L}$ or IL-1 $\beta$ at $10 \mathrm{ng} / \mathrm{mL}$ induced phosphorylation of NF- $\kappa B$ p65 (Fig. 5A, B). These results suggest that NF- $\kappa B$ is activated in response to MIA or IL- $1 \beta$ stimulation in chondrocytes. Pretreatment with nicotine at 10 $\mu \mathrm{mol} / \mathrm{L}$ suppressed IL-1 $\beta$ - or MIA-induced increases in phosphorylated NF- $\kappa \mathrm{B}$ p 65 , and these effects could be prevented by $10 \mathrm{nmol} / \mathrm{L}$ MLA (Fig. 5C, D). Nicotine or MLA administration alone did not significantly affect NF- $\kappa B$ p65 phosphorylation in chondrocytes ( $P>0.05$ compared with controls, data not shown). This data point to nicotine acting, via $\alpha 7$-nAChRs, dampens phosphorylation of NF- $\kappa$ B p65.

\section{Discussion}

Both the initiation and advancement of $\mathrm{OA}$ are now thought to include inflammation even in the preliminary stages of the disease [27]. Several studies show a distinct connection 
Fig. 5. The effects of nicotine on MIA- or IL1 $\beta$-induced NF- $\kappa$ B p65 activation in chondrocytes. $(A, B)$ Chondrocytes were treated with $10 \mu \mathrm{M}$ MIA or $10 \mathrm{ng} /$ mL IL1 $\beta$ for various lengths of time as indicated. (C,D) Chondrocytes were pretreated with nicotine $(10 \mu \mathrm{M})$ in the absence or presence of MLA (10 $\mathrm{nM}$ ) for $30 \mathrm{~min}$ and then challenged with MIA $(10 \mu \mathrm{M})$ or IL1 $\beta$ (10 $\mathrm{ng} / \mathrm{mL}$ ) for $30 \mathrm{~min}$. After treatment, chondrocytes were harvested and levels of phosphorylated NF- $\mathrm{BB}$ p65 were analyzed. Lower: representative blots are shown. Upper: densitometric analysis of the phosphorylated forms of NF- $\kappa B$ p65. Data are presented as the mean \pm SEM of four independent experiments. ${ }^{*} P<$ 0.05 and ${ }^{* *} P<0.01$ vs. the control (saline alone) group; $\# P<0.05$ and $\# \# P<0.01$ vs. the IL1 $\beta$ treatment group. ${ }^{*} P<0.05$ and $* * P<0.01$ between the IL1 $\beta+$ nicotine treatment group and the IL1 $\beta+$ nicotine + MLA treatment group.

between the evolution of tibiofemoral cartilage damage and the occurrence of activated synovial macrophages $[28,29]$. Discharged inflammatory factors such as proinflammatory cytokines are essential mediators of the disturbed metabolism and enhanced catabolism of joint tissue involved in OA [6]. Consequently, drugs that can inhibit the performance of secreted inflammatory molecules, such as proinflammatory cytokines, and the ensuing inflammatory progression may contribute to articular fortification.

During this investigation, we found that systemic administration of nicotine significantly improved MIA-induced cartilage degradation assessed by macroscopic and Mankin's score of knee joints. Additionally, we discovered that nicotine inhibited MIA- or IL1 $\beta$-stimulated elevation of phosphorylation of p38, Erk1/2 and JNK MAPKs and of NF- $\kappa$ B p65 from reactive chondrocytes. The p38, Erk1/2 and JNK MAPKs signaling pathways are essential in cartilage depletion [30]. Sondergaard et al. found that inhibition of the MAPK p38, p44/42 and Src family abolished proteolytic cartilage degradation by blocking MMP synthesis and activity [31]. Moreover, growing evidence demonstrates that NF- $\kappa B$ signaling as not only playing a key role in the pro-inflammatory stress-related responses of chondrocytes to extra- and intra-cellular insults, but also in the management of their differentiation program [32]. Martel-Pelletier et al. found that MAPKs and NF- $\kappa B$ together regulate interleukin-17-induced nitric oxide production in human osteoarthritic chondrocytes [33]. Taken together, our data indicate that the p38, Erk1/2 and JNK MAPKs and NF- $\mathrm{BB}$ signaling-transduction pathways are involved in the inhibitory effects of nicotine on MIA- or IL1 $\beta$-induced chondrocyte activation.

The Framingham study was one of the first to generate the hypothesis that smoking may protect against OA. The negative association was identified in both cross-sectional [34] and cohort analyses [35]. This supposition is also well advocated by several epidemiological reports [36-40], which have reported that the frequency of OA is lower in smokers than in 
nonsmokers, leading to the hypothesis that the reduced occurrence of OA in smokers may be due to the presence of nicotine from tobacco. Considerable evidence supports that nicotine exerts its effects primarily by acting on nAChRs [41]. These subunits combine in the formation of a large family of nAChRs, the most abundant of which are heteromeric $\beta 2$-containing nAChRs and homomeric $\alpha 7$ nAChRs. Recent studies suggest that the role of $\alpha 7$-nAChRs in immune responses and inflammatory cascades has attracted much interest according to their apparent relevance to a variety of human diseases, such as diabetes, asthma, cystic fibrosis, Parkinson's disease, sepsis, ulcerative colitis and arthritis. Those diseases have been related to runaway cytokine-mediated inflammation [42]. Cytokine-based therapeutic agents play a major role in the treatment of these diseases. The 'nicotinic anti-inflammatory pathway' may have critical clinical implications, as treatment with nicotinic agonists can potentially moderate the production of pro-inflammatory cytokines from immune cells via $\alpha 7$ homomeric nAChRs ( $\alpha 7-n A C h R s)[43,44]$.

In the current study, we found that the alleviation of MIA-induced cartilage degradation in the knee was reversed by the $\alpha 7$-nAChR-selective antagonist MLA. In rat primary cultured chondrocytes, pretreatment with nicotine suppressed MIA- or IL1 $\beta$-induced chondrocyte activation, as shown by inhibition of phosphorylation of p38, Erk1/2 and JNK MAPKs and NF- $\kappa B$ p65. These inhibitory effects of nicotine were also reversed by MLA. Those data demonstrated that nicotine-induced protection in a MIA rat model of OA occurs via $\alpha 7$-nAChR-mediated inhibition of chondrocytes. It is of interest to explore the possible mechanisms by which $\alpha 7$-nAChRs could trigger responses in different cells. At first, it was thought that the cell response was mediated by influx of calcium through activated nAChRs, causing an increase in the intracellular calcium concentration, and then activated variety signaling cascade. Moreover, $\alpha 7-n A C h R$ agonists also can modulate several other signaling pathways such as Jak2/STAT3 signaling pathway [45]. Together with results from various other studies, these data suggest that additional and yet not fully clear mechanisms to link ligand gated ion channels to intracellular signaling pathways might exist [41].

However, nicotine might also play a much more direct part in this process. Data from a previous study showed that nicotine upregulates glycosaminoglycan and collagen synthesis by articular chondrocytes at physiological levels seen in those who smoke [46]. Ying et al. reported that nicotine promoted proliferation and collagen synthesis of chondrocytes isolated from normal human and OA patients [47]. The exact mechanism underlying the protection provided by nicotine must still be fully characterized.

Our results are the first to demonstrate that nicotine inhibits MIA-induced (in vivo) and MIA- or IL1 $\beta$-induced (in vitro) chondrocyte activation via $\alpha 7$-nAChRs and decreases the resulting phosphorylation of p38, Erk1/2 and JNK MAPKs and NF- $\mathrm{B}$ p65. These data strongly support the hypothesis that inhibition of chondrocyte activation by stimulation of $\alpha 7$-nAChRs may provide a new therapeutic strategy for treatment of inflammation-related disorders. Thus, nicotinic agonists that specifically target the $\alpha 7$-nAChR might serve as potential therapeutic agents for $\mathrm{OA}$ and other inflammation-related diseases.

\section{Abbreviations}

OA (osteoarthritis); $\alpha 7$-nAChR ( $\alpha 7$ nicotinic acetylcholine receptor); MIA (monosodium iodoacetate); IL (interleukin); TNF (tumor necrosis factor); AIA (adjuvant-induced arthritis); CIA (collagen-induced arthritis); MAPKs (mitogen-activated protein kinases); NF-кB (nuclear factor-kappa B); MLA (methyllycaconitine citrate); Erk1/2 (extracellular regulated kinase1/2); JNK (c-Jun-N-terminal kinase).

\section{Disclosure Statement}

The authors state that no conflicts of interest exist. 
Liu et al.: Activation of a7-nAChRs Prevents MIA-Induced Osteoarthritis

\section{Acknowledgements}

This study was supported by grants from the National Natural Science Foundation of China (No. 81373397 and 81341017), the Jiangsu Department of Science and Technology Basic Research Program (No. BK20131443), and the State Scholarship Fund (No. 201208320286). Authors also thank Dr. Devin Taylor for his assistance for edition of English writing for this paper.

\section{References}

$>_{1}$

Shen J, Chen D: Recent progress in osteoarthritis research. J Am Acad Orthop Surg 2014;22:467-468.

Elloumi M, Kallel MH: Which relationship does osteoarthritis share with smoking. Osteoarthritis Cartilage 2007;15:1097-1098.

-3 Di GD, Discacciati A, Orsini N, Wolk A: Cigarette smoking and risk of rheumatoid arthritis: a dose-response meta-analysis. Arthritis Res Ther 2014;16:R61.

4 Veenhof C, Huisman PA, Barten JA, Takken T, Pisters MF: Factors associated with physical activity in patients with osteoarthritis of the hip or knee: a systematic review. Osteoarthritis Cartilage 2012;20:6-12. Aigner T, Soder S, Gebhard PM, McAlinden A, Haag J: Mechanisms of disease: role of chondrocytes in the pathogenesis of osteoarthritis--structure, chaos and senescence. Nat Clin Pract Rheumatol 2007;3:391399.

-6 Kapoor M, Martel-Pelletier J, Lajeunesse D, Pelletier JP, Fahmi H: Role of proinflammatory cytokines in the pathophysiology of osteoarthritis. Nat Rev Rheumatol 2011;7:33-42.

7 Hui M, Doherty M, Zhang W: Does smoking protect against osteoarthritis? Meta-analysis of observational studies. Ann Rheum Dis 2011;70:1231-1237.

-8 Sugiyama D, Nishimura K, Tamaki K, Tsuji G, Nakazawa T, Morinobu A, Kumagai S: Impact of smoking as a risk factor for developing rheumatoid arthritis: a meta-analysis of observational studies. Ann Rheum Dis 2010;69:70-81.

-9 Yu H, Yang YH, Rajaiah R, Moudgil KD: Nicotine-induced differential modulation of autoimmune arthritis in the Lewis rat involves changes in interleukin-17 and anti-cyclic citrullinated peptide antibodies. Arthritis Rheum 2011;63:981-991.

- 10 van Maanen MA, Stoof SP, Larosa GJ, Vervoordeldonk MJ, Tak PP: Role of the cholinergic nervous system in rheumatoid arthritis: aggravation of arthritis in nicotinic acetylcholine receptor alpha7 subunit gene knockout mice. Ann Rheum Dis 2010;69:1717-1723.

-11 van Maanen MA, Lebre MC, van der Poll T, LaRosa GJ, Elbaum D, Vervoordeldonk MJ, Tak PP: Stimulation of nicotinic acetylcholine receptors attenuates collagen-induced arthritis in mice. Arthritis Rheum 2009;60:114-122.

12 Guingamp C, Gegout-Pottie P, Philippe L, Terlain B, Netter P, Gillet P: Mono-iodoacetate-induced experimental osteoarthritis: a dose-response study of loss of mobility, morphology, and biochemistry. Arthritis Rheum 1997;40:1670-1679.

-13 Lee SW, Song YS, Shin SH, Kim KT, Park YC, Park BS, Yun I, Kim K, Lee SY, Chung WT, Lee HJ, Yoo YH: Cilostazol protects rat chondrocytes against nitric oxide-induced apoptosis in vitro and prevents cartilage destruction in a rat model of osteoarthritis. Arthritis Rheum 2008;58:790-800.

14 Bohanon TC, Schneider RK, Weisbrode SE: Fusion of the distal intertarsal and tarsometatarsal joints in the horse using intraarticular sodium monoiodoacetate. Equine Vet J 1991;23:289-295.

-15 Wang XD, Kou XX, He DQ Zeng MM, Meng Z, Bi RY, Liu Y, Zhang JN, Gan YH, Zhou YH: Progression of cartilage degradation, bone resorption and pain in rat temporomandibular joint osteoarthritis induced by injection of iodoacetate. PLoS One 2012;7:e45036.

-16 van der Kraan PM, Vitters EL, van de Putte LB, van den Berg WB: Development of osteoarthritic lesions in mice by "metabolic" and "mechanical" alterations in the knee joints. Am J Pathol 1989;135:1001-1014.

17 Guzman RE, Evans MG, Bove S, Morenko B, Kilgore K: Mono-iodoacetate-induced histologic changes in subchondral bone and articular cartilage of rat femorotibial joints: an animal model of osteoarthritis. Toxicol Pathol 2003;31:619-624. 
Liu et al.: Activation of a7-nAChRs Prevents MIA-Induced Osteoarthritis

18 Liu Y, Hu J, Wu J, Zhu C, Hui Y, Han Y, Huang Z, Ellsworth K, Fan W: alpha7 nicotinic acetylcholine receptormediated neuroprotection against dopaminergic neuron loss in an MPTP mouse model via inhibition of astrocyte activation. J Neuroinflammation 2012;9:98.

19 Okun A, Liu P, Davis P, Ren J, Remeniuk B, Brion T, Ossipov MH, Xie J, Dussor GO, King T, Porreca F: Afferent drive elicits ongoing pain in a model of advanced osteoarthritis. Pain 2012;153:924-933.

20 Guingamp C, Gegout-Pottie P, Philippe L, Terlain B, Netter P, Gillet P: Mono-iodoacetate-induced experimental osteoarthritis: a dose-response study of loss of mobility, morphology, and biochemistry. Arthritis Rheum 1997;40:1670-1679.

-21 Mankin HJ, Dorfman H, Lippiello L, Zarins A: Biochemical and metabolic abnormalities in articular cartilage from osteo-arthritic human hips. II. Correlation of morphology with biochemical and metabolic data. J Bone Joint Surg Am 1971;53:523-537.

-22 Grossin L, Cournil-Henrionnet C, Pinzano A, Gaborit N, Dumas D, Etienne S, Stoltz JF, Terlain B, Netter P, Mir LM, Gillet P: Gene transfer with HSP 70 in rat chondrocytes confers cytoprotection in vitro and during experimental osteoarthritis. FASEB J 2006;20:65-75.

23 Okun A, Liu P, Davis P, Ren J, Remeniuk B, Brion T, Ossipov MH, Xie J, Dussor GO, King T, Porreca F: Afferent drive elicits ongoing pain in a model of advanced osteoarthritis. Pain 2012;153:924-933.

24 Malemud CJ, Islam N, Haqqi TM: Pathophysiological mechanisms in osteoarthritis lead to novel therapeutic strategies. Cells Tissues Organs 2003;174:34-48.

25 Xue J, Wang J, Liu Q, Luo A: Tumor necrosis factor-alpha induces ADAMTS-4 expression in human osteoarthritis chondrocytes. Mol Med Rep 2013;8:1755-1760.

26 Malemud CJ: Anticytokine therapy for osteoarthritis: evidence to date. Drugs Aging 2010;27:95-115.

27 Felson DT: Clinical practice. Osteoarthritis of the knee. N Engl J Med 2006;354:841-848.

28 Bondeson J: Activated synovial macrophages as targets for osteoarthritis drug therapy. Curr Drug Targets 2010;11:576-585.

-29 Bondeson J, Blom AB, Wainwright S, Hughes C, Caterson B, van den Berg WB: The role of synovial macrophages and macrophage-produced mediators in driving inflammatory and destructive responses in osteoarthritis. Arthritis Rheum 2010;62:647-657.

- 30 Saklatvala J: Inflammatory signaling in cartilage: MAPK and NF-kappaB pathways in chondrocytes and the use of inhibitors for research into pathogenesis and therapy of osteoarthritis. Curr Drug Targets 2007;8:305-313.

-31 Sondergaard BC, Schultz N, Madsen SH, Bay-Jensen AC, Kassem M, Karsdal MA: MAPKs are essential upstream signaling pathways in proteolytic cartilage degradation--divergence in pathways leading to aggrecanase and MMP-mediated articular cartilage degradation. Osteoarthritis Cartilage 2010;18:279-288.

-32 Marcu KB, Otero M, Olivotto E, Borzi RM, Goldring MB: NF-kappaB signaling: multiple angles to target OA. Curr Drug Targets 2010;11:599-613.

33 Martel-Pelletier J, Mineau F, Jovanovic D, Di BJA, Pelletier JP: Mitogen-activated protein kinase and nuclear factor kappaB together regulate interleukin-17-induced nitric oxide production in human osteoarthritic chondrocytes: possible role of transactivating factor mitogen-activated protein kinase-activated proten kinase (MAPKAPK). Arthritis Rheum 1999;42:2399-2409.

- 34 Felson DT, Anderson JJ, Naimark A, Hannan MT, Kannel WB, Meenan RF: Does smoking protect against osteoarthritis. Arthritis Rheum 1989;32:166-172.

- 35 Felson DT, Zhang Y, Hannan MT, Naimark A, Weissman B, Aliabadi P, Levy D: Risk factors for incident radiographic knee osteoarthritis in the elderly: the Framingham Study. Arthritis Rheum 1997;40:728-733.

-36 Sandmark H, Hogstedt C, Lewold S, Vingard E: Osteoarthrosis of the knee in men and women in association with overweight, smoking, and hormone therapy. Ann Rheum Dis 1999;58:151-155.

37 Cerhan JR, Wallace RB, el-Khoury GY, Moore TE: Risk factors for progression to new sites of radiographically defined osteoarthritis in women. J Rheumatol 1996;23:1565-1578.

- 38 Elloumi M, Kallel MH: Which relationship does osteoarthritis share with smoking. Osteoarthritis Cartilage 2007;15:1097-1098.

39 Hui M, Doherty M, Zhang W: Does smoking protect against osteoarthritis? Meta-analysis of observational studies. Ann Rheum Dis 2011;70:1231-1237.

40 Jarvholm B, Lewold S, Malchau H, Vingard E: Age, bodyweight, smoking habits and the risk of severe osteoarthritis in the hip and knee in men. Eur J Epidemiol 2005;20:537-542. 


\section{Cellular Physiology Cell Physiol Biochem 2015;35:627-638}

and Biochemistry

Liu et al.: Activation of a7-nAChRs Prevents MIA-Induced Osteoarthritis

41 Hurst R, Rollema H, Bertrand D: Nicotinic acetylcholine receptors: from basic science to therapeutics. Pharmacol Ther 2013;137:22-54.

42 Bencherif M, Lippiello PM, Lucas R, Marrero MB: Alpha7 nicotinic receptors as novel therapeutic targets for inflammation-based diseases. Cell Mol Life Sci 2011; 68: 931-949.

43 Ulloa L: The vagus nerve and the nicotinic anti-inflammatory pathway. Nat Rev Drug Discov 2005;4:673684.

44 Olofsson PS, Rosas-Ballina M, Levine YA, Tracey KJ: Rethinking inflammation: neural circuits in the regulation of immunity. Immunol Rev 2012;248:188-204.

45 de Jonge WJ, Ulloa L: The alpha7 nicotinic acetylcholine receptor as a pharmacological target for inflammation. Br J Pharmacol 2007;151: 915-929.

46 Gullahorn L, Lippiello L, Karpman R: Smoking and osteoarthritis: differential effect of nicotine on human chondrocyte glycosaminoglycan and collagen synthesis. Osteoarthritis Cartilage 2005;13:942-943.

-47 Ying X, Cheng S, Shen Y, Cheng X, An RF, Wang W, Lin Z, Chen Q Zhang W, Kou D, Peng L, Tian XQ, Lu CZ: Nicotine promotes proliferation and collagen synthesis of chondrocytes isolated from normal human and osteoarthritis patients. Mol Cell Biochem 2012;359:263-269. 